\title{
Fluorescence signatures of SARS-CoV-2 spike S1 proteins and an human ACE-2: excitation-emission maps and fluorescence lifetimes
}

\author{
Jonas Grzesiak $^{\mathrm{a}}$, Lea Fellner ${ }^{\mathrm{a}}$, Karin Grünewald ${ }^{\mathrm{a}}$, Christoph Kölbl ${ }^{\mathrm{a}}$, Arne Walter ${ }^{\mathrm{a}}$, \\ Reinhold Horlacher $^{\mathrm{b}}$, Frank Duschek ${ }^{\mathrm{a}}$ \\ ${ }^{a}$ German Aerospace Center (DLR), Institute of Technical Physics, Im Langen Grund 1, 74239 Hardthausen, Germany \\ ${ }^{\text {b}}$ Trenzyme GmbH, Byk-Gulden-Str. 2, 78467 Konstanz, Germany
}

\begin{abstract}
.
Significance: Fast and reliable detection of infectious SARS-CoV-2 virus loads is an important issue. Fluorescence spectroscopy is a sensitive tool to do so in clean environments. This presumes a comprehensive knowledge of fluorescence data.

Aim: This work aims at providing fully featured information on wavelength and time-dependent data of the fluorescence of the SARS-CoV-2 spike protein S1 subunit, its receptor binding domain (RBD) and the human angiotensinconverting enzyme 2 (hACE2), especially with respect to possible optical detection schemes.

Approach: Spectrally resolved excitation-emission maps of the involved proteins and measurements of fluorescence lifetimes were recorded for excitations from 220 to $295 \mathrm{~nm}$. The fluorescence decay times were extracted by using a bi-exponential kinetic approach. The binding process in the SARS-CoV-2 RBD was likewise examined for spectroscopic changes.

Results: Distinct spectral features for each protein are pointed out in relevant spectra extracted from the excitation emission maps. We also identify minor spectroscopic changes under the binding process. The decay times in the bi-exponential model are found to be $(2.0 \pm 0.1) \mathrm{ns}$ and $(8.0 \pm 1.0) \mathrm{ns}$.

Conclusions: Specific material data serve as important background information for the design of optical detection and testing methods for SARS-CoV-2 loaded media.
\end{abstract}

Keywords: excitation-emission maps, fluorescence decay times, fluorescence, SARS-CoV-2, angiotensin-converting enzyme 2 .

*Jonas Grzesiak, tp-apw@dlr.de

\section{Introduction}

The global spread of the SARS-CoV-2 virus, which started in early 2020 [1] and which was declared pandemic by the WHO in March 2020 [2], has promoted the intensive research on human coronaviruses [3], which are a global public health thread [4]. A major tool in monitoring and controlling the spread of the viruses is a fast, accurate and sensitive detection of the virus or the infection[5]. In the case of SARS-CoV-2, a significant number of research articles on sampling techniques, nano biosensor technologies and antigen testing have been published and are reviewed e.g. in Refs. [6], [7, 8] and [9], respectively. An overview on photonic approaches for detecting 
virus loads and infections by optical means is given in Ref. [10]. The authors summarize that spectroscopic techniques, such as FTIR, Raman and fluorescence, are still facing challenges from the huge diversity of viruses in the human organism. Among those methods, laser induced fluorescence (LIF) is a widely used tool to investigate proteins [11, 12, 13, 14]. Elastic light scattering and fluorescence have been used to observe virus particles and virus-like particles $[15,16,17]$. The LIF technology takes advantage of increased signal intensities and has been applied to detect and classify viruses with excitation in the near UV [18, 19]. As pointed out in Ref. [20] , additional, orthogonal information on fluorescence characteristics can be retrieved not only from two-dimensional, spectral signatures (excitation-emission maps) but also from fluorescence decay times. Such distinct features are of high importance for a reliable sample classification[21].

In this work, high resolution fluorescence data of the proteins involved in the SARS-CoV-2 binding process, namely the S1 part of the spike protein of SARS-CoV-2, the receptor-binding domain thereof and human angiotensin-converting enzyme 2 (hACE2) are reported as well as results on their fluorescence decay times. Spectral data and fluorescence decay times of the investigated proteins are compared and discussed as candidates for classification features.

\section{Experimental setup}

The proteins used for this study are SARS-CoV-2 S1 RBD (S1 RBD domain with His-Tag, $\mathrm{M}=27.5 \mathrm{kDa}$ ), SARS-CoV-2 S1 (S1 domain with His-tag, 77.1 kDa) and hACE2 (ECD domain, tag free processes $\mathrm{M}=80 \mathrm{kDa}$ ). The proteins were produced by trenzyme $\mathrm{GmbH}$ using a transient production system in HEK293 suspension cells followed by purification either via the encoded His-Tag by IMAC (RBD and S1 protein) or by ion exchange chromatography (hACE2). After purification, the buffer of the purified proteins was exchanged to DPBS (Dulbecco's phosphate 
buffered saline (DPBS), $\mathrm{pH}=7.1-7.5$, Sigma-Aldrich) and the proteins were analyzed. The final concentrations of the solutions were $22.6 \mu \mathrm{g} / \mathrm{ml}$ in case of the hACE2, $7.8 \mu \mathrm{g} / \mathrm{mlin}$ the RBD case and $22.2 \mu \mathrm{g} / \mathrm{ml}$ for the full length $\mathrm{S} 1$ protein. This ensures comparable, molar concentrations of the proteins for the analysis.

The samples were investigated in UV-transparent cuvettes (fused silica glass, Hellma 117F), with a light travel path of $10 \mathrm{~mm}$ in the spectrometer.

A FS5 fluorescence spectrometer from Edinburgh Instruments was used to obtain full fluorescence signatures of the proteins. The spectrometer uses a $90^{\circ}$ setup and a variable wavelength excitation. Additionally it allows for time correlated single photon measurements of the samples. The fluorescence maps contain data for excitation wavelengths ranging from $220 \mathrm{~nm}-300 \mathrm{~nm}$ and emission from $230 \mathrm{~nm}-500 \mathrm{~nm}$. Time dependent fluorescence signals have been recorded at an excitation at $260 \mathrm{~nm}$, with $0.9 \mathrm{~ns}$ excitation pulse duration and the respective optimal emission wavelengths.

\section{Results and Discussion}

\subsection{LIF spectral signatures of the hACE2 enzyme and the S1 protein}

As an overview, the emission maps of the SARS-CoV-2 S1 spike protein, the receptor-binding domain (RBD) thereof and the hACE2 are shown in Fig. 1. The diagonal trace of peaks, at $+3430 \mathrm{~cm}^{-1}$ from the excitation wavelengths $\lambda_{e x}$ are assigned to be the Raman signals of $\mathrm{H}_{2} \mathrm{O}$. The narrow peaks in the lower right corner of the graphs are assigned to be the second order diffraction maximum. Each map consists of two major maxima: the first one located at an excitation wavelength $\lambda_{e x}=220 \mathrm{~nm}$ with maximum emission at $\lambda_{e m}=325 \mathrm{~nm}$ for S1 and hACE2 and 

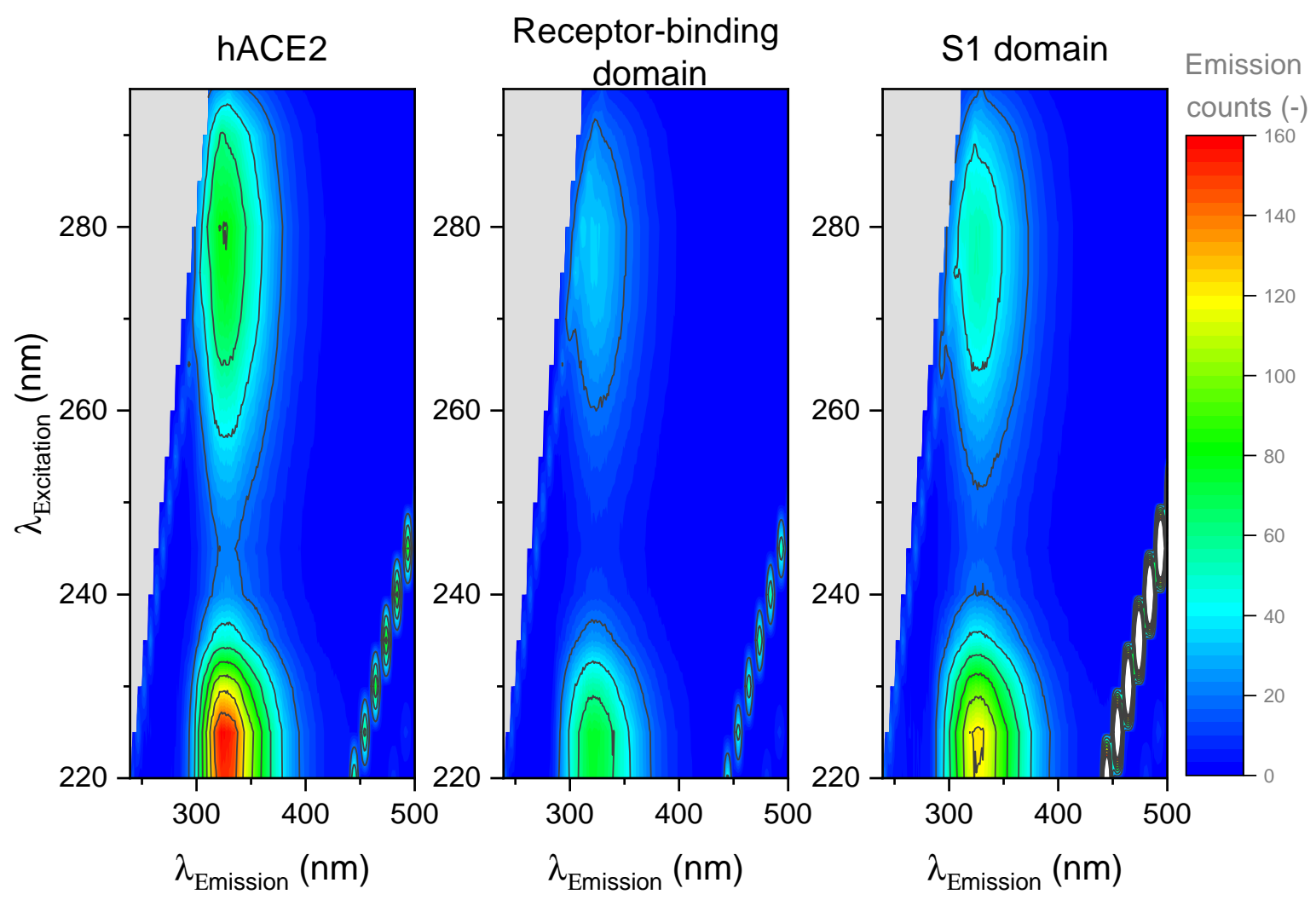

Fig 1 Excitation-emission fluorescence map for hACE2 (left), the receptor-binding domain (center) and SARS-CoV-2 S1 (right). Data are available on biorxiv.org as supplementary material.

at $\lambda_{e m}=320 \mathrm{~nm}$ for the receptor-binding domain. The second major maximum appears at an excitation wavelength of $\lambda_{e x}=280 \mathrm{~nm}$ showing a maximum emission at $\lambda_{e m}=325 \mathrm{~nm}$ for all three proteins.

Fig. 2 displays the normalized fluorescence response of the three samples in detail at excitation wavelengths $\lambda_{e x}=220 \mathrm{~nm}$ and $\lambda_{e x}=280 \mathrm{~nm}$. For clearness the emission spectra obtained at $\lambda_{e x}=280 \mathrm{~nm}$ have been adjusted by the background signal from a pure PBS sample. The signals at $\lambda_{e x}=220 \mathrm{~nm}$ clearly show the Raman signal of the solvent as well as the second order diffraction maximum. The intensities of the respective signals are indicated by the Raman signal intensities and are more explicit in Fig. 3. The fluorescence peaks are located between $290 \mathrm{~nm}$ and $410 \mathrm{~nm}$, which indicates that the excitation light mainly probes tryptophane and tyrosine sites 
inside the proteins. At both respective excitation wavelengths, the three proteins show significantly different fluorescence responses. This is manifested in differently shaped peaks, which in turn give full width half maximum values of the peaks from $55 \mathrm{~nm}$ for hACE2 and the receptor-binding domain to $60 \mathrm{~nm}$ for the $\mathrm{S} 1$ protein, both at an excitation wavelength of $\lambda_{e x}=280 \mathrm{~nm}$. The same pattern can be seen for $\lambda_{e x}=220 \mathrm{~nm}$, with slightly broader values for full width at half maximum (FWHM): $58 \mathrm{~nm}$ for hACE2 and RBD and $63 \mathrm{~nm}$ for the S1 protein. These slightly broader spectra may emerge from an excited hot (i.e. vibrationally excited) state with broad emission spectrum due to the lower excitation wavelength.
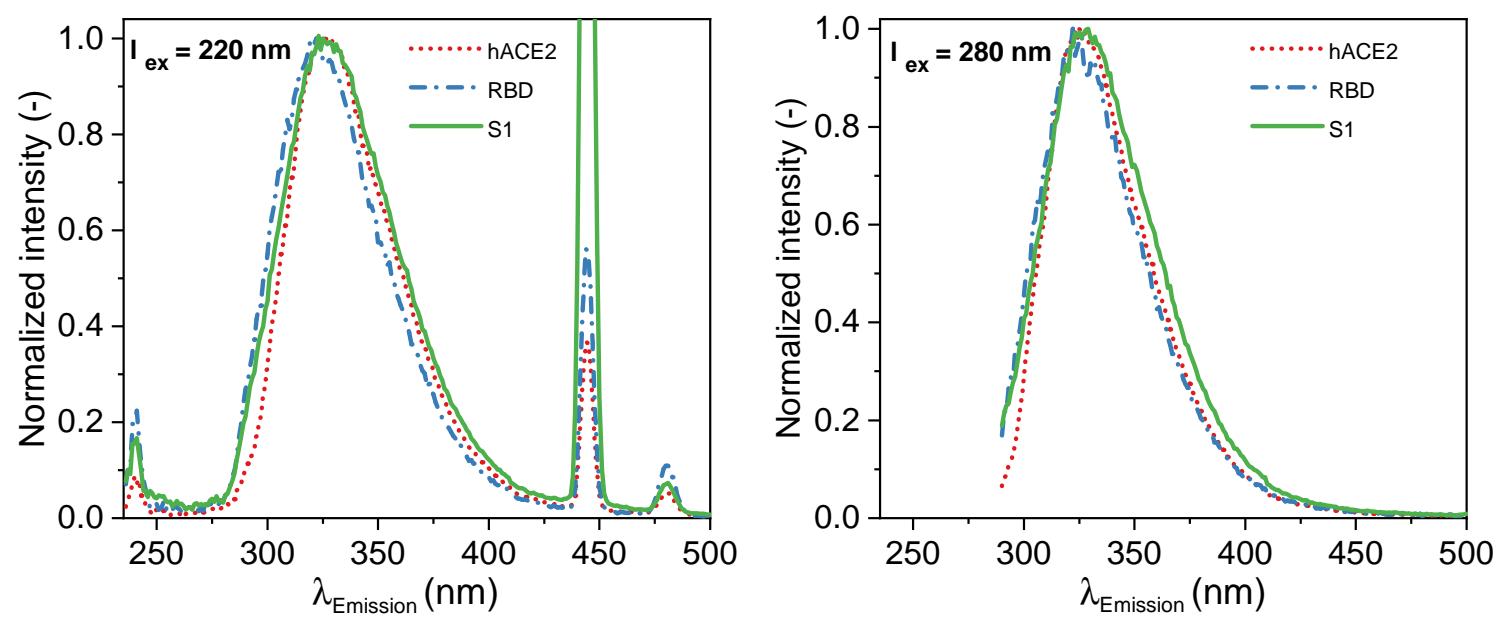

Fig 2 Detailed, normalized fluorescence signals of the three proteins at $\lambda_{e x}=220 \mathrm{~nm}$ (left) and $\lambda_{e x}=280 \mathrm{~nm}$ (right).

Besides the pure protein signals, also the bound complex of the virus with its receptor is of interest for a possible virus detection. To obtain a protein-enzyme complex solution we combined both the solution of the S1 full length protein and the RBD protein solution with the hACE2 solution: $1 \mathrm{ml}$ of each solution with the respective concentrations noted above to ensure equimolarity of both substances in the combined solution. Straight after combination, the mixture was stirred to ensure a fully mixed solution. From the receptor binding affinity of about 1-40 nM [22] and the concentration of the $\mathrm{S} 1$ protein and the RBD protein analytes of about $3.0 \cdot 10^{-7} \frac{\mathrm{Mol}}{\mathrm{l}}$ one ob- 
tains a half life time of the binding process in the range of $\tau \approx 3-35 \mathrm{~s}$. Thus, the full binding of all hACE2 receptors to the virus proteins in our solution is ensured within $5 \mathrm{~min}$, when the spectra were taken. Fig. 3 shows the fluorescence results of the bound complexes at an excitation wavelength of $280 \mathrm{~nm}$. The signals have been adjusted by the background signal from a pure PBS sample and for simplicity the normalized signals are shown. For comparison the single contributions are shown too. We compared the signal of the bound complexes with the sum of the signals of the single contributions (black dashed lines in the spectra). The difference spectra between the summed signals and the bound complexes are shown in Fig. 4. Here, the significant change of about $-1 \%$ of the signal at $312 \mathrm{~nm}$ is due to differences in the $\mathrm{H}_{2} \mathrm{O}$ Raman signal. At $350-400$ $\mathrm{nm}$ however, a slight change in the slopes of the peak is found, indicating a possible conformation change under the binding process, especially in the S1 case.
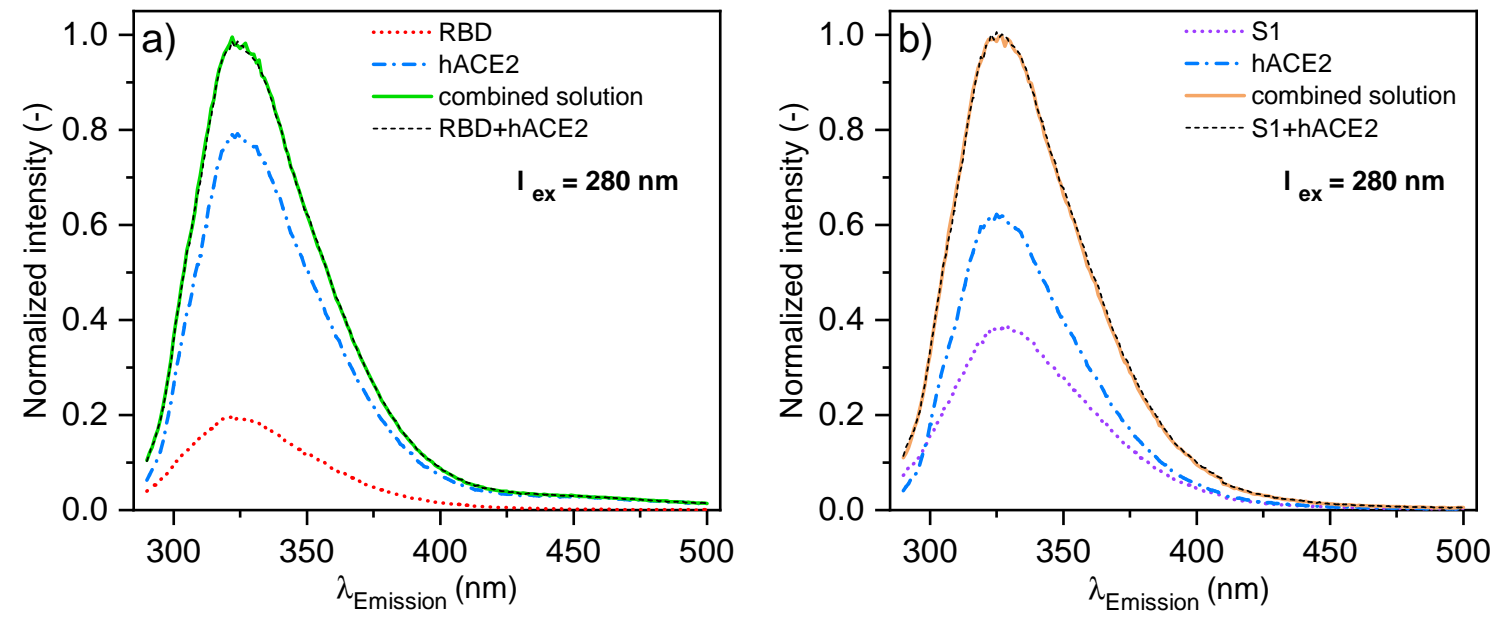

Fig 3 Fluorescence spectra of bound protein complexes and their single contributions: a) Receptor-binding domain of the $S 1$ protein and $b$ ) the full $S 1$ protein.

\subsection{Fluorescence decay times}

Additionally, the fluorescence decay of the base solutions of the hACE2, the RBD protein and the S1 protein as well as of their bound complex solutions were measured at an excitation of 260 


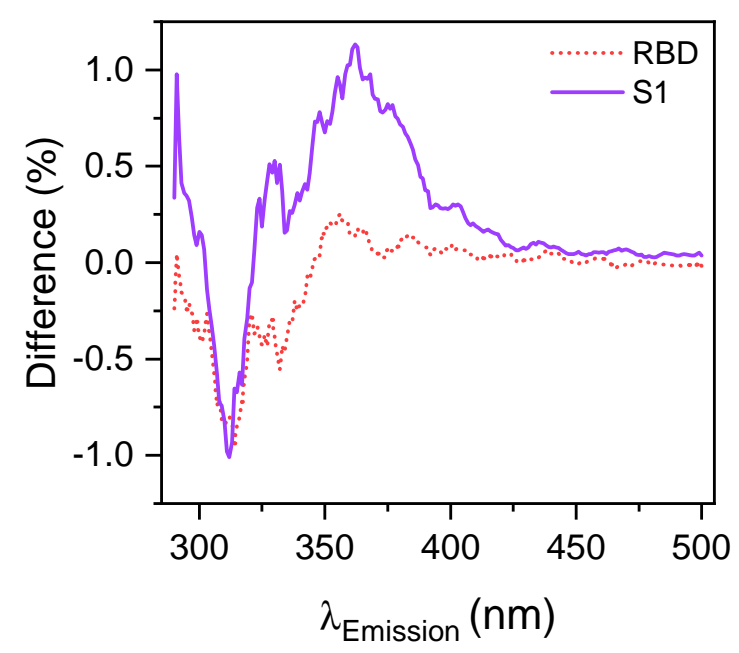

Fig 4 Differences between the added single contributions and the bound complex for the RBD and the S1 case.

\begin{tabular}{|c|l|l|}
\hline & $\tau_{1}(\mathrm{~ns})$ & $\tau_{2}(\mathrm{~ns})$ \\
\hline hACE2 & $2.0 \pm 0.1$ & $8.0 \pm 1.4$ \\
\hline RBD & $1.9 \pm 0.1$ & $9.0 \pm 1.9$ \\
\hline S1 & $2.1 \pm 0.1$ & $6.9 \pm 1.5$ \\
\hline
\end{tabular}

Table 1 Results of the double exponential fit to the different protein time correlated single photon signals at an excitation wavelength of $260 \mathrm{~nm}$.

$\mathrm{nm}$ as a time correlated single photon counting signal, shown in Fig. 5. The decay signals were evaluated in the well established double exponential decay model [23, 24]. Here, the fits were performed as global fits, where all signals are taken into account at once and the fit for the bound complexes is fitted as a sum of the single contributions. Results of the fits are summarized in Table 1, the error noted is the error due to the fit routine. For both global fits the coefficient of determination is $\mathrm{R}^{2}=0.99$. The fit analysis for the decays yields that the two time constants in the exponential decays, $\tau_{1}$ and $\tau_{2}$, are only slightly different for all proteins and bound proteincomplexes. The recorded signals consist of a fast and a slow decay with $\tau_{1} \approx(2.0 \pm 0.1) \mathrm{ns}$ and $\tau_{2} \approx(8.0 \pm 1.0) \mathrm{ns}$ for all proteins. 

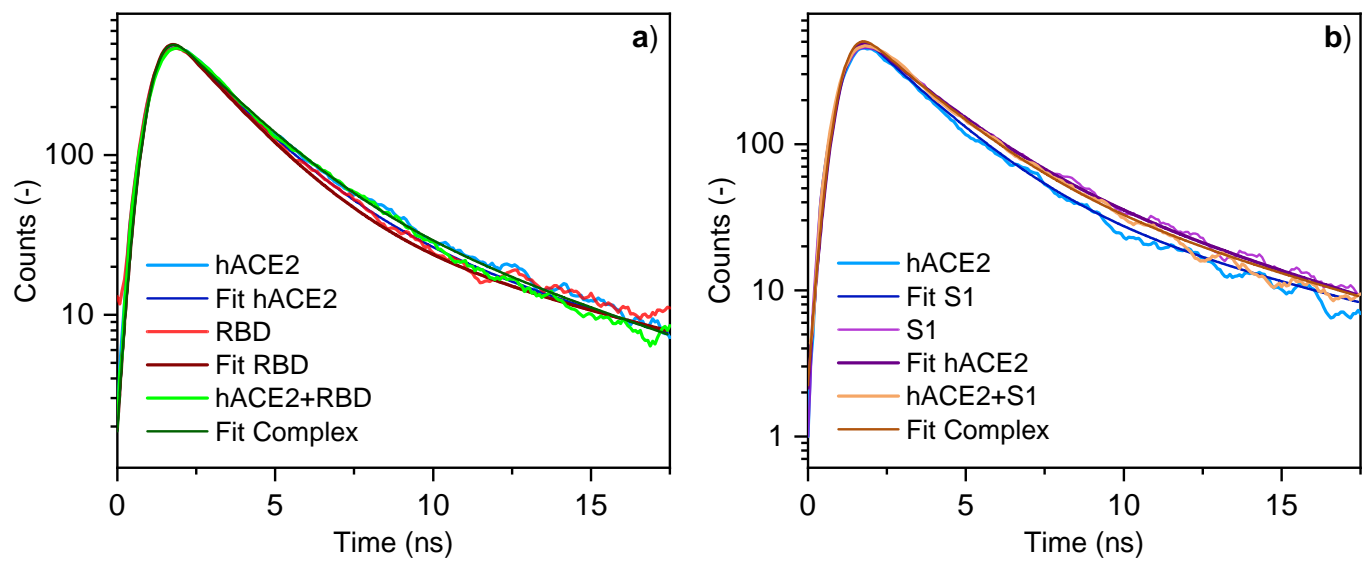

Fig 5 Time correlated single photon counting signals for the different solutions and their respective double exponential decay fit. a) for the RBD case, b) for the $\mathrm{S} 1$ case.

\section{Summary}

We have presented fluorescence data for the S1 spike protein of the SARS-CoV-2, its receptorbinding domain and for its most important receptor in human organisms, hACE2. The spectra show distinguishable features such as slight, but clear shifts in the shapes of the LIF signatures for all proteins, e.g. manifested in different FWHMs of the respective fluorescence peaks. Additionally, the signals vary with the excitation wavelength and show for example a clear blueshift of about $5 \mathrm{~nm}$ for the receptor-binding domain at $\lambda_{e x}=220 \mathrm{~nm}$ in contrast to $\lambda_{e x}=280 \mathrm{~nm}$. The fluorescence decay times have been determined to be $\tau_{1} \approx(2.0 \pm 0.1)$ ns and $\tau_{2} \approx(8.0 \pm 1.0)$ ns. For further insight, the limitations are the signal to noise ratios and the maximum possible integration times in order to avoid photolytic decomposition of the proteins in the UV. The data are publicly available for external $R \& D$ as described in section 4.

\section{Disclosures}

The authors declare no financial or ethical conflicts of interest. 
Data and Materials Availability

Measurement data will be available on biorxiv.org.

\section{References}

[1] P. Zhou, X.-L. Yang, X.-G. Wang, et al., "A pneumonia outbreak associated with a new coronavirus of probable bat origin," Nature 579, 270-273 (2020).

[2] D. Cucinotta and M. Vanelli, "WHO Declares COVID-19 a Pandemic," Acta Bio Medica Atenei Parmensis 91, 157-160 (2020).

[3] M. Haghani and M. C. J. Bliemer, "Covid-19 pandemic and the unprecedented mobilisation of scholarly efforts prompted by a health crisis: Scientometric comparisons across SARS, MERS and 2019-nCov literature," Scientometrics 125, 2695-2726 (2020).

[4] E. de Wit, N. van Doremalen, D. Falzarano, et al., "SARS and MERS: recent insights into emerging coronaviruses," Nature Reviews Microbiology 14, 523-534 (2016).

[5] G. Guglielmi, "Rapid coronavirus tests: a guide for the perplexed," Nature 590, 202-205 (2021).

[6] A. Mohammadi, E. Esmaeilzadeh, Y. Li, et al., "SARS-CoV-2 detection in different respiratory sites: A systematic review and meta-analysis,” EBioMedicine 59, 102903 (2020).

[7] A. D. Teklemariam, M. Samaddar, M. G. Alharbi, et al., "Biosensor and molecular-based methods for the detection of human coronaviruses: A review," Molecular and Cellular Probes 54, 101662 (2020). 
[8] B. Alhalaili, I. N. Popescu, O. Kamoun, et al., "Nanobiosensors for the detection of novel coronavirus 2019-nCoV and other pandemic/epidemic respiratory viruses: A review, sars," Sensors 20, 6591 (2020).

[9] T. Toptan, L. Eckermann, A. E. Pfeiffer, et al., "Evaluation of a SARS-CoV-2 rapid antigen test: Potential to help reduce community spread?," Journal of Clinical Virology 135, 104713 (2021).

[10] J. Lukose, S. Chidangil, and S. D. George, "Optical technologies for the detection of viruses like COVID-19: Progress and prospects," Biosensors and Bioelectronics 178, 113004 (2021).

[11] J. R. Lakowicz, Ed., Principles of Fluorescence Spectroscopy, Springer US (2006).

[12] L. R. Dartnell, T. A. Roberts, G. Moore, et al., "Fluorescence characterization of clinicallyimportant bacteria," PLoS ONE 8, e75270 (2013).

[13] M. S. Ammor, "Recent advances in the use of intrinsic fluorescence for bacterial identification and characterization," J Fluoresc 17, 455-459 (2007).

[14] S. Cabredo, A. Parra, and J. Anzano, "Bacteria spectra obtained by laser induced fluorescence," Journal of Fluorescence 17, 171-180 (2007).

[15] A. Alimova, A. Katz, R. Podder, et al., "Virus particles monitored by fluorescence spectroscopy: A potential detection assay for macromolecular assembly," Photochemistry and Photobiology 80, 41-46 (2004).

[16] M. Fang, W. Diao, B. Dong, et al., "Detection of the assembly and disassembly of PCV2b 
virus-like particles using fluorescence spectroscopy analysis," Intervirology 58(5), 318-323 (2015).

[17] M. Rüdt, P. Vormittag, N. Hillebrandt, et al., "Process monitoring of virus-like particle reassembly by diafiltration with UV/vis spectroscopy and light scattering," Biotechnology and Bioengineering 116, 1366-1379 (2019).

[18] B. S, G. JL, B. M, et al., "Non-contact, real-time laser-induced fluorescence detection and monitoring of microbial contaminants on solid surfaces before, during and after decontamination," Journal of Biosensors \& Bioelectronics 09(02) (2018).

[19] V. Gabbarini, R. Rossi, J.-F. Ciparisse, et al., "Laser-induced fluorescence (LIF) as a smart method for fast environmental virological analyses: validation on picornaviruses," Scientific Reports 9, 1-7 (2019).

[20] F. Gebert, M. Kraus, L. Fellner, et al., "Novel standoff detection system for the classification of chemical and biological hazardous substances combining temporal and spectral laserinduced fluorescence techniques $\star$," The European Physical Journal Plus 133, 1-5 (2018).

[21] M. Carestia, R. Pizzoferrato, M. Gelfusa, et al., "Development of a rapid method for the automatic classification of biological agents' fluorescence spectral signatures," Optical Engineering 54, 114105 (2015).

[22] Y. M. Bar-On, A. Flamholz, R. Phillips, et al., "SARS-CoV-2 (COVID-19) by the numbers," eLife 9 (2020).

[23] K. Willis, A. Szabo, J. Drew, et al., "Resolution of heterogeneous fluorescence into compo- 
nent decay-associated excitation spectra. application to subtilisins," Biophysical Journal 57, $183-189$ (1990).

[24] A. G. Szabo and D. M. Rayner, "Fluorescence decay of tryptophan conformers in aqueous solution," Journal of the American Chemical Society 102, 554-563 (1980).

\section{List of Figures}

1 Excitation-emission fluorescence map for hACE2 (left), the receptor-binding domain (center) and SARS-CoV-2 S1 (right). Data are available on biorxiv.org as supplementary material.

2 Detailed, normalized fluorescence signals of the three proteins at $\lambda_{e x}=220 \mathrm{~nm}$ (left) and $\lambda_{e x}=280 \mathrm{~nm}$ (right).

3 Fluorescence spectra of bound protein complexes and their single contributions: a) Receptor-binding domain of the S1 protein and b) the full S1 protein.

4 Differences between the added single contributions and the bound complex for the RBD and the S1 case.

5 Time correlated single photon counting signals for the different solutions and their respective double exponential decay fit. a) for the RBD case, b) for the S1 case.

\section{List of Tables}

1 Results of the double exponential fit to the different protein time correlated single photon signals at an excitation wavelength of $260 \mathrm{~nm}$. 